\title{
Hermitization and the Poisson bracket-commutator correspondence as a consequence of averaging
}

\author{
Adriana I Pesci ${ }^{1}$, Raymond E Goldstein ${ }^{1,2}$ and Hermann Uys ${ }^{1}$ \\ ${ }^{1}$ Department of Physics, University of Arizona, Tucson, AZ 85721, USA \\ ${ }^{2}$ Program in Applied Mathematics, University of Arizona, Tucson, AZ 85721, USA \\ E-mail: pesci@physics.arizona.edu, gold@physics.arizona.edu and uys@physics.arizona.edu
}

Received 18 March 2005, in final form 27 October 2005

Published 11 January 2006

Online at stacks.iop.org/JPhysA/39/789

\begin{abstract}
Here we present a study of the solutions and mathematical structure of the momentum-averaged Liouville (or Collisionless Boltzmann) equation in Fourier space. We show that the averaging procedure leads to a formalism identical to that of the density matrix of quantum mechanics. This mathematical mapping leaves the averages of all quantities unaltered and provides a unique way to construct the Hermitian version of a given operator. This seems to be the only method that resolves the ambiguity of Hermitization of operators that contain products of non-commuting variables. We also present a systematic perturbation scheme to evaluate correctly the classical solutions from the quantum ones and a formal proof of the approximate correspondence between the Poisson brackets and commutators.
\end{abstract}

PACS numbers: $03.65 . \mathrm{Ta}, 05.20 . \mathrm{Dd}, 45.05 .+\mathrm{x}, 03.65 . \mathrm{Sq}$

\section{Introduction}

Taking the classical limit of an arbitrary quantum system is more an art than a systematic procedure. Even apparently simple problems present serious difficulties [1]. For example, when showing the correspondence between commutators and Poisson brackets it is necessary to deal with the ambiguity present in the construction of a Hermitian operator. Then, there is the passage from quantum probabilities to probabilities in phase space which has seen through the years a wealth of attempts to create a suitable representation of the quantum distribution function in phase space. The best known and most successful example of such an attempt is the Wigner function, even though it is a well-known fact that it gives rise to negative probabilities in many cases [2]. As if all these difficulties were not enough, taking the limit $\hbar \rightarrow 0$ in Schrödinger's equation is at best a singular procedure, rendering very difficult any attempt to construct a perturbation theory. 
There are other rather intriguing facts that do not seem to have a very good explanation. For linear systems the time evolution of observables in quantum mechanics can be described by the corresponding average values obtained from the probability density function in phase space calculated from Liouville's equation [3, 4]. Furthermore, the equation that governs the Wigner function corresponds exactly to the Liouville equation for all potentials with vanishing derivatives of order three or larger [2]. This remarkable connection between the Wigner function and its classical counterpart has lead to its widespread use within so-called decoherence theory. The aim of such theories is to describe the process by which a system existing in a coherent quantum mechanical superposition of states loses its coherence so that it comes to obey classical probability distributions. This is often phrased as 'the transition from quantum to classical world'. A widely accepted picture, views this process of 'decoherence' as the result of entanglement between the system under study and an environment not monitored by the observer (for extensive reviews see [5-7]). The aim of the present paper is not to contradict such theories, for indeed many of their predictions have been the subject of careful experimental scrutiny [8-11]. What connections there exist between these theories and our interpretation of the 'transition from classical to quantum' is not entirely clear. We do wish to note, however, that the point of view described below would see as distinct the processes of 'loss of coherence' and the 'transition from quantum to classical'. For example, an electron in a hydrogen atom might exist in a superposition of the first and second excited states. Decoherence theory will describe the process by which the electron will come to be in one state or the other with classical probabilities. On the other hand, one may want to explore the possibility that there is a way in which the solutions to the Schrödinger equation can be adjusted as one scales the problem to the macroscopic level, that is, the transition from quantum orbitals to an ensemble of particles following deterministic trajectories.

Here, we propose an unorthodox way to think about these problems which sheds some light on the issues discussed above. The procedure that we outline is based on the previous work which describes a map of the first two moments of the non-relativistic Boltzmann equation onto the quantum fluid equations [12-14] that lead to either the Schrödinger or Pauli equations for irrotational or vortical flows, respectively, [15, 16]. An analogous mapping was introduced for the relativistic Boltzmann equation and in this case one obtains either the Klein-Gordon or the second-order Dirac equation [17].

We start by performing the mapping using only the first moment of the Boltzmann equation. Note that this mapping would give the same result if applied to the Liouville equation because taking averages of the first two moments in the momentum coordinate cancels out the collision integral of the Boltzmann equation, rendering the results identical to those obtained in the collisionless case. Through this procedure, we obtain a series expansion in the variable $\mathbf{y}$ conjugate to the momentum. In the limit $\mathbf{y} \rightarrow 0$, we obtain the equation that governs the evolution of the Wigner function and hence the standard Schrödinger equation for the wavefunction and its conjugate, thus establishing an unequivocal relationship between the classical and Wigner distribution functions. From this derivation, it becomes clear why the probability distribution must be the product of the wavefunction and its conjugate, why the linear combinations of wavefunctions that correspond to coherent states are the closest to the classical picture, and also why the Wigner function looks like a symmetrized Fourier anti-transform. This expansion also sets the stage for a perturbation theory that allows us to recover the classical limit up to the order of approximation desired.

To understand the nature of quantum averages we introduce classical averages and then transform them according to the prescription of the mapping. In the limit $\mathbf{y} \rightarrow 0$, we find that the transformed averages coincide exactly with the averages prescribed by the postulates of quantum mechanics up to the accuracy of the wavefunction. In fact, in the 
new representation, the classical momentum has acquired the form of the momentum operator of quantum mechanics (!). The only exception to this correspondence occurs in situations in which the quantities to be averaged involve products of conjugate variables (e.g., $x p_{x}$ ), in which case the corresponding quantum average has a new 'operator' that happens to be one of the many possible Hermitian versions [1] of the original quantity. This method establishes a preferred way to perform the Hermitization procedure. The equality between the quantum and the classical averages is as exact as the wavefunction, i.e. there are no further approximations introduced beyond the limit $\mathbf{y} \rightarrow 0$ to obtain the wavefunction.

Finally, we map the Poisson brackets onto the commutators. In this case we show that the correspondence between these two quantities is far from exact and in particular deviates considerably for any operators that have a dependence on the momentum of higher degree than a second power. This also agrees with the known fact that the Poisson brackets correspond to commutators up to powers of $\hbar^{2}$ [1]. This discrepancy and its correction are illustrated in a detailed example presented.

\section{The mapping}

It is well known that the motion of an ensemble of $N$ classical particles governed by Liouville's equation can be recast in a hierarchy of non-linear partial differential equations (PDEs) for the reduced probability functions defined as follows:

$$
f_{N}\left(\mathbf{x}_{1}, \mathbf{p}_{1}, \ldots, \mathbf{x}_{N}, \mathbf{p}_{N}\right)=\frac{D}{\int_{\Omega} D \mathrm{~d} \Omega},
$$

and for $1 \leqslant j<N$

$$
f_{j}\left(\mathbf{x}^{j}, \mathbf{p}^{j}\right)=\int_{\Omega} f_{N}\left(\mathbf{x}^{N}, \mathbf{p}^{N}\right) \prod_{l=j+1}^{N} \mathrm{~d} \mathbf{x}_{l} \mathrm{~d} \mathbf{p}_{l},
$$

where $D$ represents the number density of points in phase space, $\Omega$ is the volume in phase space and $\left(\mathbf{x}^{N}, \mathbf{p}^{N}\right)=\left(\mathbf{x}_{1}, \mathbf{p}_{1}, \ldots, \mathbf{x}_{N}, \mathbf{p}_{N}\right)$. These functions correspond to the probability of finding the subsystem of $j<N$ particles in the phase volume $\prod_{l=1}^{j} \mathrm{~d} \mathbf{x}_{l} \mathrm{~d} \mathbf{p}_{l}$ about the state $\left(\mathbf{x}_{1}, \mathbf{p}_{1}, \ldots, \mathbf{x}_{j}, \mathbf{p}_{j}\right)$. The $N$ PDEs generated are known as the BBKGY hierarchy [18], the first two members of which (i.e., the equations for $f_{1}$ and $f_{2}$ ) are used to determine the kinetic and potential energy of an aggregate of particles, and have a crucial role in fluid dynamics. One way to solve these equations is to decouple them through an ansatz with regard to the properties of the functions $f_{j}$. When this Bogoliubov ansatz is imposed, the resulting equation for $f_{1}=f_{1}\left(\mathbf{x}_{1}, \mathbf{p}_{1}, t\right)$ is the Boltzmann equation

$$
\frac{\partial f_{1}}{\partial t}+\frac{\mathbf{p}_{1}}{m} \cdot \frac{\partial f_{1}}{\partial \mathbf{x}_{1}}+\mathbf{F}\left(\mathbf{x}_{1}, \mathbf{p}_{1}\right) \cdot \frac{\partial f_{1}}{\partial \mathbf{p}_{1}}=\int \mathrm{d} \mathbf{x}_{2} \int \mathrm{d} \mathbf{p}_{2}\left[f_{1}\left(\mathbf{p}_{1}^{\prime}\right) f_{1}\left(\mathbf{p}_{2}^{\prime}\right)-f_{1}\left(\mathbf{p}_{1}\right) f_{1}\left(\mathbf{p}_{2}\right)\right],
$$

where $\mathbf{F}$ is the external force averaged over all other coordinates.

Suppose now that we do not have a good way to obtain the exact initial conditions of particle 1 . We can only tell that the area of phase space must be conserved so that the system will remain a Hamiltonian one. We can try to perform an average over the coordinates while at the same time enforcing the constraint of area conservation. The hope is that this procedure would lead to an averaged equation of motion with a consistent statistics as is the case with the equations of fluid mechanics where the concept of particle trajectories is replaced by that of streamlines. Nothing would be new in this scheme were it not that we propose to do all the 
work in Fourier space. This is not done in the usual way by transforming in $\mathbf{x}_{1}$, but instead by transforming in $\mathbf{p}_{1}$ as if we were anti-transforming back to an original coordinate $\mathbf{y}$. This set of transformations will be faithful to the original problem only if we can reintroduce the two central properties of area preservation and of conjugate character of position and momentum. Without them the system would lose its Hamiltonian nature. In what follows we will see that this seems to be possible.

We first write formally the average of the Boltzmann equation over the coordinates in phase space. It is of interest to note that when the Boltzmann equation is integrated over the momentum coordinate $\mathbf{p}_{1}$ it produces the conservation law for the number of particles, when multiplied by $\mathbf{p}_{1}$ and integrated over $\mathbf{p}_{1}$ it gives the momentum balance equation, and when multiplied by $\mathbf{p}_{1}^{2}$ it gives the energy balance equation after integration over the momentum. Moreover, the collision integral cancels out in all three cases [18]. Thus, the result for our first balance equation reads

$$
\int_{-\infty}^{+\infty} \mathrm{d} \mathbf{x} \mathrm{d} \mathbf{p}\left(\frac{\partial f}{\partial t}+\frac{\mathbf{p}}{m} \cdot \frac{\partial f}{\partial \mathbf{x}}-\frac{\partial V(\mathbf{x})}{\partial \mathbf{x}} \cdot \frac{\partial f}{\partial \mathbf{p}}\right)=0,
$$

where we have dropped the subindex 1 from $f_{1}, \mathbf{x}_{1}$ and $\mathbf{p}_{1}$ and also have assumed only conservative forces and used the identity $\mathbf{F}=-\partial V / \partial \mathbf{x}$ derived from Hamilton's equations of motion. It should be pointed out here that the integration over the $\mathbf{x}$ variable is in reality unnecessary, however it will be useful later on to motivate a canonical change of variables necessary for the transformation.

We now introduce into (4) the following representation for $f$ :

$$
f(\mathbf{x}, \mathbf{p}, t)=\frac{1}{(2 \pi \eta)^{3}} \int_{-\infty}^{+\infty} \exp \left(-\mathrm{i} \frac{\mathbf{p} \cdot \mathbf{y}}{\eta}\right) \hat{f}(\mathbf{x}, \mathbf{y}, t) \mathrm{d} \mathbf{y},
$$

where $\hat{f}(\mathbf{x}, \mathbf{y}, t)$ is of course given by

$$
\hat{f}(\mathbf{x}, \mathbf{y}, t)=\int_{-\infty}^{+\infty} \exp \left(\mathrm{i} \frac{\mathbf{p} \cdot \mathbf{y}}{\eta}\right) f(\mathbf{x}, \mathbf{p}, t) \mathrm{d} \mathbf{p} .
$$

The constant $\eta$ is the only free parameter of the theory. In principle it could be adjusted through experimental measurement. It will become clear that enforcing $\eta=\hbar$ leads to quantum mechanics, however for the sake of generality we will keep the notation $\eta$. With these definitions and some straightforward algebra, equation (4) can be written as [15]

$$
\int_{-\infty}^{+\infty} \mathrm{d} \mathbf{x} \mathrm{d} \mathbf{y} \delta(\mathbf{y}) \frac{\mathrm{i}}{\eta}\left[\frac{\eta}{\mathrm{i}} \frac{\partial \hat{f}}{\partial t}-\frac{\eta^{2}}{m} \frac{\partial}{\partial \mathbf{x}} \cdot \frac{\partial \hat{f}}{\partial \mathbf{y}}+\mathbf{y} \cdot \frac{\partial V}{\partial \mathbf{x}} \hat{f}\right]=0
$$

where we have made use of the convergence of the function $\hat{f}$ to discard the surface terms resulting from an integration by parts.

This new version of Boltzmann's equation, in which we have taken the average over $\mathbf{p}$, has introduced in the system an important change. The original equation had to be solved in conjunction with Hamilton's equations in order to find meaningful solutions. Of particular importance is $\mathrm{d} p_{j} / \mathrm{d} t=-\partial H / \partial x_{j}$ for each $j$ component of the momentum, which can be written with finite differences as $\Delta p_{j} \Delta x_{j}=-\Delta H \Delta t$. Now, due to the introduction of the Fourier transform we have enforced a lower bound, equal to $\eta / 2$, to the product $\Delta p_{j} \Delta x_{j}$ which is the area in phase space. Thus the equations of motion in finite differences can be written as

$$
\Delta p_{j} \Delta x_{j}=-\Delta H \Delta t=\frac{\eta}{2},
$$

leading to the conclusion that of all possible solutions we might be able to generate for our averaged Boltzmann equation in Fourier space, the only ones faithful to the classical Hamiltonian system will be those for which the equality is enforced. 
With this in mind, following Fröhlich [19-21], we now introduce the canonical change of variables $\mathbf{y}=\mathbf{x}^{\prime}-\mathbf{x}^{\prime \prime}$ and $\mathbf{x}=\left(\mathbf{x}^{\prime}+\mathbf{x}^{\prime \prime}\right) / 2$, in order to find an expression of (7) more amenable to solution. This change of variables satisfies

$$
\begin{aligned}
& \mathbf{x}^{\prime}=\mathbf{x}+\frac{\mathbf{y}}{2}, \quad \mathbf{x}^{\prime \prime}=\mathbf{x}-\frac{\mathbf{y}}{2}, \\
& \frac{\partial}{\partial \mathbf{y}}=\frac{1}{2}\left(\frac{\partial}{\partial \mathbf{x}^{\prime}}-\frac{\partial}{\partial \mathbf{x}^{\prime \prime}}\right), \quad \frac{\partial}{\partial \mathbf{x}}=\left(\frac{\partial}{\partial \mathbf{x}^{\prime}}+\frac{\partial}{\partial \mathbf{x}^{\prime \prime}}\right) .
\end{aligned}
$$

Equation (7) then becomes

$$
\begin{aligned}
& \int_{-\infty}^{+\infty} \mathrm{d} \mathbf{x}^{\prime} \mathrm{d} \mathbf{x}^{\prime \prime} \delta\left(\mathbf{x}^{\prime}-\mathbf{x}^{\prime \prime}\right)\left[\frac{\eta}{\mathrm{i}} \frac{\partial \hat{f}}{\partial t}-\frac{\eta^{2}}{2 m}\left(\frac{\partial^{2} \hat{f}}{\partial \mathbf{x}^{\prime 2}}-\frac{\partial^{2} \hat{f}}{\partial \mathbf{x}^{\prime \prime 2}}\right)+\left(V\left(\mathbf{x}^{\prime}\right)-V\left(\mathbf{x}^{\prime \prime}\right)\right) \hat{f}\right] \\
&+\mathcal{O}\left(\left(\mathbf{x}^{\prime}-\mathbf{x}^{\prime \prime}\right)^{3}\right)=0,
\end{aligned}
$$

where we have used the identity

$\mathbf{y} \cdot \frac{\partial V}{\partial \mathbf{x}}=V\left(\mathbf{x}+\frac{\mathbf{y}}{2}\right)-V\left(\mathbf{x}-\frac{\mathbf{y}}{2}\right)-\sum_{\left\{n_{i}\right\}}^{\infty} \frac{2}{n_{1} ! n_{2} ! n_{3} !} \frac{\partial^{n_{1}+n_{2}+n_{3}} V}{\partial x_{1}^{n_{1}} \partial x_{2}^{n_{2}} \partial x_{3}^{n_{3}}}\left(\frac{y_{1}}{2}\right)^{n_{1}}\left(\frac{y_{2}}{2}\right)^{n_{2}}\left(\frac{y_{3}}{2}\right)^{n_{3}}$,

and $\left(x_{1}, x_{2}, x_{3}\right) \equiv \mathbf{x},\left(y_{1}, y_{2}, y_{3}\right) \equiv \mathbf{y}$ and $\left\{n_{i}\right\}$ indicates the set of indices $n_{1}, n_{2}, n_{3}$ which are constrained so that $n_{1}+n_{2}+n_{3}=2 n+1 \geqslant 3$ is an odd number. Note that we have chosen to use a symmetrized difference to replace the derivative. The reason for this choice is twofold: first, as is well known from numerical analysis, errors in derivatives are smaller when the centre difference is used. Second and more important, as we have shown elsewhere [15, 16] and pointed out in introduction, there is an alternative way to obtain Schrödinger's equation and both methods should be compatible with each other. That other procedure consists of taking the first three moments of the Boltzmann (or Liouville) equation. The first two moments (conservation of points density and momentum) lead to the Schrödinger equation, and the last moment (conservation of energy) is automatically satisfied because the equation is enslaved to the other two. As a result of this calculation, any corrections must be of order $\mathcal{O}\left(\mathbf{y}^{3}\right)$ or higher, such that the second and third order moments, which involve two derivatives with respect to $\mathbf{y}$, will not require any more information about $\hat{f}$ and the potential than that included in the previous moments.

If now we evaluate the integrals in (10) the corrections vanish and we are left with two possible choices for the zeroth order term: either we take the limit $\mathbf{x}^{\prime} \rightarrow \mathbf{x}^{\prime \prime} \equiv \mathbf{x}$ which leads to the trivial solution, or we find a function $\hat{f}$ that satisfies the equation inside the square brackets in (10). Here we choose the second route, and since the equation to solve is linear we propose using the separation of variables $\hat{f}=\psi\left(\mathbf{x}^{\prime}\right) \phi\left(\mathbf{x}^{\prime \prime}\right)$. As intuitive a form as this may be, it should be emphasized that it is by no means the most general form of the solution. As with any partial differential equation, initial conditions and boundary conditions may invalidate such solutions. The results discussed in the remainder of this paper hold only under this restrictive form of solution. This leads to the following pair of equations:

and

$$
-\frac{\eta^{2}}{2 m} \frac{\partial^{2} \psi\left(\mathbf{x}^{\prime}\right)}{\partial \mathbf{x}^{\prime 2}}+V\left(\mathbf{x}^{\prime}\right) \psi\left(\mathbf{x}^{\prime}\right)=-\frac{\eta}{\mathrm{i}} \frac{\partial \psi\left(\mathbf{x}^{\prime}\right)}{\partial t}
$$

$$
-\frac{\eta^{2}}{2 m} \frac{\partial^{2} \phi\left(\mathbf{x}^{\prime \prime}\right)}{\partial \mathbf{x}^{\prime \prime 2}}+V\left(\mathbf{x}^{\prime \prime}\right) \phi\left(\mathbf{x}^{\prime \prime}\right)=\frac{\eta}{\mathrm{i}} \frac{\partial \phi\left(\mathbf{x}^{\prime \prime}\right)}{\partial t},
$$

where we have set the separation constant equal to zero, a choice which corresponds to fixing a particular zero of the potential. Since (13) is the conjugate of (12) it follows that, 
in the limit $\mathbf{x}^{\prime} \rightarrow \mathbf{x}^{\prime \prime} \equiv \mathbf{x}, \phi(\mathbf{x})=\psi^{\dagger}(\mathbf{x})$ and then $\hat{f}=\psi^{\dagger} \psi$ where $\left(^{\dagger}\right)$ stands for complex conjugation. Any general solution can now be written as a linear combination of these basis functions. Finally, to obtain the approximate solution to the original equation in phase space we anti-transform $\hat{f}$. For the case of a particular basis function product, the anti-transform yields:

$$
\begin{aligned}
f(\mathbf{x}, \mathbf{p}, t) & =\int_{-\infty}^{+\infty} \mathrm{d}\left(\mathbf{x}^{\prime}-\mathbf{x}^{\prime \prime}\right) \mathrm{e}^{-\frac{\mathrm{i}}{\eta} \mathbf{p} \cdot\left(\mathbf{x}^{\prime}-\mathbf{x}^{\prime \prime}\right)} \hat{f}\left(\mathbf{x}^{\prime}, \mathbf{x}^{\prime \prime}, t\right) \\
& =\int_{-\infty}^{+\infty} \mathrm{d} \mathbf{y} \mathrm{e}^{-\frac{\mathrm{i}}{\eta} \mathbf{p} \cdot \mathbf{y}} \psi\left(\mathbf{x}-\frac{\mathbf{y}}{2}\right) \psi^{\dagger}\left(\mathbf{x}+\frac{\mathbf{y}}{2}\right),
\end{aligned}
$$

where we have made use of the relationships (9) and $\hat{f}\left(\mathbf{x}^{\prime}, \mathbf{x}^{\prime \prime}, t\right)=\psi\left(\mathbf{x}^{\prime}\right) \psi^{\dagger}\left(\mathbf{x}^{\prime \prime}\right)$. This is the well-known Wigner function of a pure state. This first approximation to the one-particle distribution function may be a very poor one because we have never enforced the constraint (8). However, since we are dealing with a partial differential equation that is linear, it is possible, in principle, to construct a linear combination of the basis functions such that it will satisfy equation (8) at all times. A function $\hat{f}$ so constructed will be a first approximation to a proper solution of Liouville's equation, defining a positive definite probability distribution function moving along a classical trajectory. These linear combinations are known in quantum mechanics as coherent states.

By enforcing a condition on the distribution function which only holds in the limit $\mathbf{x}^{\prime} \rightarrow \mathbf{x}^{\prime \prime}$, the behaviour of the function elsewhere is at best ambiguous, rendering the limiting operation irreversible. From the derivation above, it is clear that the classical result can only be recovered if we forgo the $\mathbf{y} \rightarrow 0$ limit and devise a perturbation scheme in powers of $\mathbf{y}$ for the function $\hat{f}$. The simplest such scheme consists of proposing for $\hat{f}$ a series expansion in powers of $\mathbf{y}$. As an example, for a one-dimensional case, we can write

$$
\hat{f}=\hat{f}_{0}+y^{4} \hat{f}_{4}+\cdots
$$

where the first correction is of fourth order in $y$ because the transformation is, as we have mentioned before, exact up to order $\mathbf{y}^{3}$ as a consequence of the symmetrization we chose when approximating the potential. We must point out that $\hat{f}_{0}$ itself depends on $y$ in a non-trivial manner; it is as if we would have summed up a class of terms into $\hat{f}_{0}$ and we are now trying to calculate the corrections to those terms. An alternative point of view on the form of these corrections comes from the mapping of kinetic equations onto the hydrodynamic formulation of quantum mechanics [15-17]. That mapping utilizes only the first three moments of the underlying kinetic distribution function, and therefore corrections naturally start at fourth order. When the expression (15) is replaced into (10) without the truncation at third order, i.e. including all the terms in expression (11), we obtain for the zeroth order the usual pair of equations (12) and (13), after using separation of variables. For the first-order correction in the one-dimensional case, we obtain

$$
\frac{16(3 !) \eta^{2}}{m}\left(\frac{\partial \hat{f}_{4}}{\partial x^{\prime}}+\frac{\partial \hat{f}_{4}}{\partial x^{\prime \prime}}\right)+\left(\frac{\partial}{\partial x^{\prime}}+\frac{\partial}{\partial x^{\prime \prime}}\right)^{3} V\left(\frac{x^{\prime}+x^{\prime \prime}}{2}\right) \psi^{\dagger}\left(x^{\prime}\right) \psi\left(x^{\prime \prime}\right)=0
$$

which is, in principle, non-separable. This lack of separability is also true at all other orders, making the task of solving the hierarchy all the more difficult. Another difficulty is the dissimilarity between the equations generated for each successive order (see appendix A). It is possible, in principle, to find the corrections up to the order desired in a systematic way. 


\section{Averages and the Hermitization procedure}

As has been shown previously by Kaniadakis [22] and subsequently verified using a different approach [15], the classical averages reduce to the quantum ones in quite a natural manner for quantities of the form $h(\mathbf{x})+g(\mathbf{p})$, where $h$ and $g$ are analytic functions. In general,

$$
\begin{aligned}
\left\langle\mathbf{x}^{n}\right\rangle & =\frac{\int \mathbf{x}^{n} f(\mathbf{x}, \mathbf{p}, t) \mathrm{d} \mathbf{x} \mathrm{d} \mathbf{p}}{\int f(\mathbf{x}, \mathbf{p}, t) \mathrm{d} \mathbf{x} \mathrm{d} \mathbf{p}} \\
& =\frac{\int \mathbf{x}^{n}\left(\lim _{\mathbf{y} \rightarrow 0} \hat{f}\right) \mathrm{d} \mathbf{x}}{\int\left(\lim _{\mathbf{y} \rightarrow 0} \hat{f}\right) \mathrm{d} \mathbf{x}} \\
& =\frac{\int \psi^{\dagger} \mathbf{x}^{n} \psi \mathrm{d} \mathbf{x}}{\int \psi^{\dagger} \psi \mathrm{d} \mathbf{x}}
\end{aligned}
$$

and

$$
\begin{aligned}
\left\langle\mathbf{p}^{n}\right\rangle & =\frac{\int \mathbf{p}^{n} f(\mathbf{x}, \mathbf{p}, t) \mathrm{d} \mathbf{x} \mathrm{d} \mathbf{p}}{\int f(\mathbf{x}, \mathbf{p}, t) \mathrm{d} \mathbf{x} \mathrm{d} \mathbf{p}} \\
& =\frac{\int \psi^{\dagger}(-\mathrm{i} \eta \nabla)^{n} \psi \mathrm{d} \mathbf{x}}{\int \psi \psi^{\dagger} \mathrm{d} \mathbf{x}},
\end{aligned}
$$

where it is possible to make the correspondence $\mathbf{p} \equiv \hat{\mathbf{p}}=-\mathbf{i} \eta \boldsymbol{\nabla}[15,22]$. Since any analytic function can be expressed as a unique power series expansion, any expression of the form $\hat{A}=h(\mathbf{x})+g(\mathbf{p})$ where $h$ and $g$ are analytic will have an average given by [15]

$$
\begin{aligned}
\langle\hat{A}\rangle & =\frac{\int \hat{A} f(\mathbf{x}, \mathbf{p}, t) \mathrm{d} \mathbf{x} \mathrm{d} \mathbf{p}}{\int f(\mathbf{x}, \mathbf{p}, t) \mathrm{d} \mathbf{x} \mathrm{d} \mathbf{p}} \\
& =\frac{\int \psi^{\dagger} \hat{A}(\mathbf{x}, \hat{\mathbf{p}}) \psi \mathrm{d} \mathbf{x}}{\int \psi \psi^{\dagger} \mathrm{d} \mathbf{x}}
\end{aligned}
$$

This is also true for cases in which the variables $\mathbf{x}$ and $\mathbf{p}$ are mixed in products in such a way that there are no products of conjugate pairs, as with $L_{z}=x p_{y}-y p_{x}$. These kinds of combinations have the property that their quantum counterparts are Hermitian and it is possible to make the direct correspondence $A_{c l}(\mathbf{x}, \mathbf{p}) \rightarrow \hat{A}(\mathbf{x},-\mathrm{i} \eta \nabla)$. On the other hand, combinations involving products of variables that are conjugate in phase space produce non-Hermitian operators when the correspondence is applied directly. It is possible to produce a Hermitian version of any such operator, but there is no unique way to do it and different Hermitizations will produce different results for the averages [1]. Here, instead of applying this 'correspondence' directly, we apply the mapping to the original classical quantity. For the sake of simplicity, we consider a single pair of conjugate variables, let us say $x$ and $p_{x} \equiv p$. Then, a typical analytic function of these variables can be written as $B(x, p)=\sum_{n, m} B_{n m} x^{n} p^{m}$. After some lengthy algebra (see appendix B), we find

$$
\begin{aligned}
\langle B(x, p)\rangle & =\frac{\int B(x, p) f(x, p, t) \mathrm{d} x \mathrm{~d} p}{\int f(x, p, t) \mathrm{d} x \mathrm{~d} p} \\
& =\frac{\int \psi^{\dagger} \hat{B}_{H}(\hat{x}, \hat{p}) \psi \mathrm{d} x}{\int \psi \psi^{\dagger} \mathrm{d} x}
\end{aligned}
$$


where

$$
\begin{aligned}
\hat{B}_{H} & =\sum_{n, m} B_{n m} \frac{1}{2^{m}} \sum_{k=0}^{m}\left(\begin{array}{l}
m \\
k
\end{array}\right) \hat{p}^{k}\left(\hat{x}^{n} \hat{p}^{m-k}\right) \\
& =\sum_{n, m} B_{n m} \frac{1}{2^{n}} \sum_{k=0}^{n}\left(\begin{array}{l}
n \\
k
\end{array}\right) \hat{x}^{n-k}\left(\hat{p}^{m} \hat{x}^{k}\right) .
\end{aligned}
$$

Here, we have used the notation $\hat{x}=x$ and $\hat{p} \equiv-\mathrm{i} \eta \partial / \partial x$, together with the identity proven by McCoy [23] (see also appendix B). The quantity $\hat{B}_{H}$ happens to be Hermitian, and is the only possible choice that satisfies the requirement of being equal to the classical average up to the accuracy of the wavefunction. Thus, this method of approaching the Hermitization procedure has the advantage of uniqueness and gives us the correspondence

$$
x^{n} p^{m} \leftrightarrow \sum_{n, m} B_{n m} \frac{1}{2^{n}} \sum_{k=0}^{n}\left(\begin{array}{l}
n \\
k
\end{array}\right) \hat{x}^{n-k}\left(\hat{p}^{m} \hat{x}^{k}\right),
$$

which is the correspondence obtained by using the operator ordering rule first introduced by Weyl [24].

We should keep in mind that the classical and 'quantum' averages are identical up to the accuracy of the separability of $\hat{f}$. Given the connection between the operations $\partial / \partial \mathbf{y}$ and multiplication by $\mathbf{p}$ it is clear that the separability of $\hat{f}$ will only hold for averages involving powers of $\mathbf{p}$ less than or equal to three. In such cases, since the corrections to $\hat{f}_{0}$ are order $\mathcal{O}\left(\mathbf{y}^{4}\right)$ or higher, as indicated in (15), taking at most three derivatives with respect to $\mathbf{y}$ leaves a correction $\mathcal{O}(\mathbf{y})$ to the average which vanishes as $\mathbf{y} \rightarrow 0$. For any special cases, where all corrections $\hat{f}_{k}(k \geqslant 4)$ to $\hat{f}_{0}$ vanish independent of the value of $\mathbf{y}$, expression (19) is exact for any power of p. This could be an explanation of the shortcomings of Weyl's rule. Shewell [25] found that following Weyl's rule the operator $\hat{H}^{2}$ corresponding to $H^{2}$, where $H=p^{2} / 2 m+(1 / 4) a x^{4}$, does not commute with the energy $H$, thus it is not a constant of the motion. This result erroneously implies energy dispersion for energy characteristic states. If we accept for the moment that quantum mechanics can be considered as a third moment kinetic theory for $p$, and since $\hat{H}^{2}$ involves a fourth power of $p$ through an average, it seems reasonable that Weyl's rule does not give the exact result.

It is noteworthy that within this scheme it is possible to obtain a different Hermitian operator, the one corresponding to the symmetrization rule

$$
x^{n} p^{m} \leftrightarrow \frac{1}{2}\left(\hat{x}^{n} \hat{p}^{m}+\hat{p}^{m} \hat{x}^{n}\right) .
$$

To obtain this other Hermitization rule we must use for the potential term a different approximation than (11). In fact, rule (22) results from using a biased difference $\left(\mathbf{x}^{\prime}=\mathbf{x}, \mathbf{x}^{\prime \prime}=\right.$ $\mathbf{x}-\mathbf{y}$ ) instead of the centre difference used in (9). The error for the biased difference, which of course vanishes in the limit $\mathbf{y} \rightarrow 0$, is $\mathcal{O}\left(\mathbf{y}^{2}\right)$. Since we want a theory exact up to the third moment, so that it will be compatible with the results we found for the hydrodynamic picture, we need the lowest corrections to be $\mathcal{O}\left(\mathbf{y}^{3}\right)$ or higher. This makes the Weyl rule the better choice.

Unlike the path integral formalism and other standard methods [26, 27], the kinetic theory approach reveals that the different Hermitization rules are associated with the different levels of accuracy of approximations in the underlying theory.

We conjecture that within this scheme it is also possible to obtain the Born-Jordan Hermitization rule [28], although we have not yet been able to do so. Thus, we cannot make any comparisons between it and Weyl's rule and the statement that Weyl's rule is the better choice is conditional. 


\section{Poisson brackets}

In this last section we apply the averaging procedure to the Poisson bracket of any two functions of $\mathbf{x}$ and $\mathbf{p}$ and study its connection to the commutator between the corresponding operators. The average of the Poisson bracket of any two quantities $u$ and $v$ is

$$
\langle\{u, v\}\rangle=\int \mathrm{d} \mathbf{x} \mathrm{d} \mathbf{p}\{u, v\} f(\mathbf{x}, \mathbf{p}, t) .
$$

We can now write $f$ in terms of $\hat{f}$ using equation (5) to obtain

$$
\langle\{u, v\}\rangle=\int \frac{\mathrm{d} \mathbf{x} \mathrm{d} \mathbf{p} \mathrm{d} \mathbf{y}}{(2 \pi \eta)^{3}}\{u, v\} \exp \left(-\mathrm{i} \frac{\mathbf{p} \cdot \mathbf{y}}{\eta}\right) \hat{f}(\mathbf{x}, \mathbf{y}, t) .
$$

For the trivial case in which the Poisson bracket is zero, the equivalence between the commutator and the bracket is obvious and of little interest. A clearly more interesting case occurs when the functions produce a non-zero bracket. To illustrate our results we will calculate the bracket for the one-dimensional case, i.e. for functions which depend only on $x, p_{x} \equiv p$ and $t$, and in particular we choose $u=u(x, t)$ and $v=p^{n}$. This choice may seem quite restrictive, but it will allow us to build the framework for the more general case. For these choices, equation (24) reads

$$
\langle\{u, v\}\rangle=\int \frac{\mathrm{d} x \mathrm{~d} p \mathrm{~d} y}{2 \pi \eta} \frac{\partial u}{\partial x} \frac{\partial v}{\partial p} \exp \left(-\mathrm{i} \frac{p y}{\eta}\right) \hat{f}(x, y, t) .
$$

After an integration by parts in $p$, replacing $v=p^{n}$ and observing that $p^{n} \mathrm{e}^{(-\mathrm{i} p y / \eta)}=$ $(-\eta / \mathrm{i})^{n}\left(\partial^{n} \mathrm{e}^{(-\mathrm{i} p y / \eta)} / \partial y^{n}\right)$, we obtain

$$
\langle\{u, v\}\rangle=\frac{\mathrm{i}}{\eta} \int \frac{\mathrm{d} x \mathrm{~d} p \mathrm{~d} y}{2 \pi \eta} y \frac{\partial u}{\partial x}\left(-\frac{\eta}{\mathrm{i}}\right)^{n} \hat{f} \frac{\partial^{n}}{\partial y^{n}} \mathrm{e}^{(-\mathrm{i} p y / \eta)} .
$$

Now we symmetrize the term $y(\partial u / \partial x)$ in the same way we did with the potential. Substituting into the expression for the average, integrating by parts $n$ times in $y$, and making use of the change of variables (9), equation (26) becomes

$$
\begin{aligned}
\langle\{u, v\}\rangle= & \int \frac{\mathrm{d} p}{2 \pi \eta} \int \mathrm{d} x^{\prime} \mathrm{d} x^{\prime \prime} \exp \left(-\mathrm{i} \frac{p\left(x^{\prime}-x^{\prime \prime}\right)}{\eta}\right)\left[\frac{1}{2^{n}}\left(\frac{\partial}{\partial x^{\prime}}-\frac{\partial}{\partial x^{\prime \prime}}\right)^{n}\left[u\left(x^{\prime \prime}\right)-u\left(x^{\prime}\right)\right] \hat{f}\right] \\
& -2(-\mathrm{i} \eta)^{n} \int \frac{\mathrm{d} p}{2 \pi \eta} \int \mathrm{d} x \mathrm{~d} y \exp \left(-\mathrm{i} \frac{p y}{\eta}\right) \sum_{k} \frac{1}{(2 k+1) !} \frac{\partial^{2 k+1} u}{\partial x^{2 k+1}} \frac{\partial^{n}}{\partial y^{n}}\left(y^{2 k+1} \hat{f}\right) .
\end{aligned}
$$

Using the binomial expression to calculate the derivatives inside the first integral, replacing $\hat{f}\left(x^{\prime}, x^{\prime \prime}, t\right)=\psi^{\dagger}\left(x^{\prime \prime}\right) \psi\left(x^{\prime}\right)$, and observing that the integral over $p$ in the first term reduces to a delta function with argument $x^{\prime}-x^{\prime \prime}$, we can calculate two of the integrals, leaving only the one over $x^{\prime} \equiv x$. Finally, integrating by parts so that $\psi^{\dagger}$ multiplies the expression from the left and $\psi$ is operated on from the left by the same expression, we obtain

$$
\begin{aligned}
\langle\{u, v\}\rangle=\frac{\mathrm{i}}{\eta} \int \mathrm{d} x \psi^{\dagger}\left[u\left(-\mathrm{i} \eta \frac{\partial}{\partial x}\right)^{n}-\left(-\mathrm{i} \eta \frac{\partial}{\partial x}\right)^{n} u\right] \psi \\
\quad-2 \frac{\mathrm{i}}{\eta} \int \mathrm{d} x \mathrm{~d} y \delta(y)(-\mathrm{i} \eta)^{n} \sum_{k=1}^{n} \frac{1}{(2 k+1) !} \frac{\partial^{2 k+1} u}{\partial x^{2 k+1}} \frac{\partial^{n}}{\partial y^{n}}\left(y^{2 k+1} \hat{f}\right) .
\end{aligned}
$$

It is useful to rewrite the derivative inside the sum by use of the binomial expansion,

$$
\sum_{k=1}^{n} \frac{1}{(2 k+1) !} \frac{\partial^{2 k+1} u}{\partial x^{2 k+1}} \sum_{\ell=0}^{n}\left(\begin{array}{l}
n \\
\ell
\end{array}\right) \frac{\partial^{\ell}}{\partial y^{\ell}} y^{2 k+1} \frac{\partial^{n-\ell}}{\partial y^{n-\ell}} \hat{f} .
$$


Since the index $k$ starts at $k=1$, for $n \leqslant 2$ the sum is $\mathcal{O}(y)$, thus the integral vanishes. On the other hand, if $n>2$ there is non-vanishing contribution proportional to $\eta^{2}$. With this in mind and noting that the first integral in (28) can be written as

$$
\begin{aligned}
\langle\{u, v\}\rangle & =\int \mathrm{d} x\left[\int \mathrm{d} p\{u, v\} f(x, p, t)\right] \\
& =\int \mathrm{d} x \psi^{\dagger}\left[\frac{\mathrm{i}}{\eta}\left[\hat{u}, \hat{p}^{n}\right]+\mathcal{O}\left(\eta^{2}\right)\right] \psi \\
& =\int \mathrm{d} x \psi^{\dagger}\left[\frac{\mathrm{i}}{\eta}[\hat{u}, \hat{v}]+\mathcal{O}\left(\eta^{2}\right)\right] \psi,
\end{aligned}
$$

we can make the correspondence

$$
\int \mathrm{d} p\{u, v\} f(x, p, t) \equiv \psi^{\dagger} \frac{\mathrm{i}}{\eta}[\hat{u}, \hat{v}] \psi+\mathcal{O}\left(\eta^{2}\right) .
$$

The correction vanishes for cases in which the variable $p$ only enters as a power less than or equal to 2, and $\hat{u}$ and $\hat{v}$ are the functions $u$ and $v$ where the momentum $p$ has been replaced by $\hat{p} \equiv-\mathrm{i} \eta(\partial / \partial x)$.

As an example, we take $u=\sin x$ and $v=p^{3}$. After a simple calculation we obtain for the commutator $[\hat{u}, \hat{v}]=(-\mathrm{i} \eta)^{2}\left(3 \sin x(\partial / \partial x)-3 \cos x\left(\partial^{2} / \partial x^{2}\right)+\cos x\right)$. If now we replace the value of the Poisson bracket $\{u, v\}=3 \cos x p^{2}$ into the average (24) and perform the integrals in $p$ and $y$ as usual, we find $(-\mathrm{i} \eta)^{2}\left(3 \sin x(\partial / \partial x)-3 \cos x\left(\partial^{2} / \partial x^{2}\right)+(3 / 4) \cos x\right)$. The discrepancy is due to the fact that $p$ enters in the calculation with a power larger than 2 . The correction as calculated from (28) is given by

$$
-\frac{\mathrm{i}}{\eta}(-\mathrm{i} \eta)^{3} \frac{1}{4} \frac{\partial^{3} u}{\partial x^{3}}=-(-\mathrm{i} \eta)^{2} \frac{1}{4} \cos x
$$

When this correction is added to the commutator we obtain the exact same result as with the Poisson bracket.

In the general case, the transformation follows identical lines except that the algebra is rather lengthy and cumbersome. We will start with two functions $u$ and $v$ dependent on all variables $x, p$ and $t$. If $u$ and $v$ are analytic functions in the variable $p$ then they can be expressed as two-power series of $p$ with coefficients that depend on $x$ and $t$

$$
u=\sum_{n=0}^{\infty} u_{n}(x, t) p^{n} \quad v=\sum_{n=0}^{\infty} v_{n}(x, t) p^{n} .
$$

The Poisson bracket of such a pair of functions is

$$
\{u, v\}=\sum_{n m} p^{n+m-1}\left(m \frac{\partial u_{n}}{\partial x} v_{m}-n \frac{\partial v_{m}}{\partial x} u_{n}\right) .
$$

The average then reduces to the sum of the averages of each term. For a suitable choice of a sequence of functions $g_{n}$, each one of these terms can be expressed in the form $n p^{n-1}(\partial g(x, t) / \partial x)$ which happens to be the Poisson bracket $\left\{g, p^{n}\right\}$.

As we saw in the previous section (see also appendix B), when we calculate the average of a non-separable function of $x$ and $p$ the result in Fourier space is the average of the corresponding Hermitian operator. The average of a Poisson bracket is no exception to the rule. This leads to a general correspondence between the Poisson bracket and the commutator of two quantities,

$$
\int \mathrm{d} p\{u, v\} f(x, p, t) \equiv \psi^{\dagger} \frac{\mathrm{i}}{\eta}\left[\hat{u}_{H}, \hat{v}_{H}\right] \psi+\mathcal{O}\left(\eta^{2}\right),
$$


where $\hat{u}_{H}$ and $\hat{v}_{H}$ are the Hermitian operator versions of the original functions $u$ and $v$. The order of the correction depends on the powers of $p$ involved. Commutators for which the highest power of $p$ is less than or equal to 2 have a vanishing correction. The coefficients of the three highest powers of $p$ obtained from the average of the Poisson bracket always coincide with the ones obtained from the commutator of the Hermitian operators. For all other powers of $p$, there are corrections that can be calculated as we have shown. This discrepancy suggests, one more time, that quantum mechanics can be thought of as a third-moment kinetic theory in the same way that classical hydrodynamics can be viewed as a second-moment kinetic theory.

It is remarkable that the most important Poisson brackets relate to functions each of which do not involve products of conjugate variables and that, moreover, involve $p$ with powers less than or equal to 2, which as we saw cancels out all corrections. Examples of this type are brackets between the angular momenta, or the canonical variables. Of particular interest is the Poisson bracket of the density matrix (which only depends on $x$ ) and the Hamiltonian (for which $x$ and $p$ enter as a sum of two functions, one of $p^{2}$ only, and the other of $x$ only) because it corresponds in commutator language to the von Neumann-Liouville equation.

\section{Conclusions}

In this work we establish the existence of a clear mathematical connection between Liouville's and Schrödinger's equations. We also provide a perturbative method to obtain corrections to the solutions to Schrödinger's equation so that it is possible to calculate the 'classical limit' in a systematic way. The same procedure applies to the Poisson brackets with relation to the commutators. We have shown that the averages of all quantities remain unchanged by the map and that as a result the Fourier representation of the original quantities provides a unique way of making the corresponding operator Hermitian.

A possible conclusion that can be drawn from our calculations is, simply stated, that Schrödinger's equation can be viewed as the equation that describes the Fourier space representation of the momentum-averaged behaviour of a set of initial conditions for a single-classical deterministic particle. If we accept that statement as the only logical consequence of the mathematical derivation we have presented in this paper, then many of the curious idiosyncrasies of quantum mechanics are revealed as a natural consequence of the transformations undergone by Liouville's equation in its reincarnation as a 'quantum' entity.

- The mysterious nature of the momentum operator as an imaginary unit times a gradient becomes an obvious consequence of working in Fourier space.

- The curious symmetrization of the density matrix that leads to the Wigner function is just the only logical and mathematically correct approximate inversion of the original transformation capable of fulfilling the constraints of conservation of points in phase space, momentum and energy.

- The 'uncertainty' principle is merely a consequence of the fact that averages undermine determinism.

- The apparent non-locality of quantum mechanics can be explained as a consequence of the averaging over all possible values of $\mathbf{p}$, since averages are, by definition, non-local.

- The averaging of initial conditions would also explain the loss of chaotic behaviour in the 'quantum' system.

- The need for Hermitization is due to the requirement of faithfulness to the classical average, with the added advantage that by following the rules derived from the classical 
origin all ambiguities are eliminated and the central role played by the Weyl ordering rule is now explained.

- As with all proper mappings, this one should preserve the mathematical structure of the original space. This explains the striking similarity between the density matrix representation of quantum mechanics and Liouville's equation in phase space, and also the 'miraculous' correspondence between the mathematical structures of classical and quantum mechanics.

- The central role of coherent states can now be understood from the constraints imposed on conjugate variables through the Fourier transform; the combinations of solutions that satisfy the equality $\Delta p \Delta x=\eta / 2$ are the only ones that should be considered as faithful transformations of the solution of Liouville's equation, and are the only ones capable of following closely the 'classical path'. All other choices will deviate considerably from the equations of motion (recall equation (8)).

Finally, looking back at the way in which the classical limit is usually obtained, we can say that taking the limit $\Delta p \Delta x=\eta / 2 \rightarrow 0$ is equivalent to the crude reinstatement of the original equations of motion. It almost seems that within the scheme presented here rather than finding the classical limit of quantum mechanics the proper question to ask should be: What corrections to quantum mechanics would reinstate the classical behaviour? The answer to that question involves calculating the correction terms that appear in the form of a perturbation theory in equation (15).

As we have pointed out, we have previously shown that it is possible to recast the first two moments of Boltzmann's equation into the corresponding quantum fluid equations that lead to Schrödinger or Pauli's equations in the non-relativistic case and to Klein-Gordon and the second-order Dirac equations in the relativistic case [15-17]. These mappings are a mathematical fact and we did not venture to guess about their possible meaning or interpretation, stubbornly clinging to the notation $\eta$ introduced by Kaniadakis [22], as opposed to $\hbar$. It seems that there is already too much interpretation surrounding quantum mechanics. However, every one of the referees for our earlier works asked the same questions: Why are we doing this mapping? and What does this mean? At the time we gave no answers, since we are not philosophers. Yet, since it seems that we cannot escape being asked these questions, we reluctantly offer the following speculation.

One possible conclusion is to consider the averages performed in the original Liouville equation as the mathematical expression of the inability to create a measurement instrument made out of parts smaller than the object to be measured, a fact for which, unfortunately, there seems to be no way around. Since the measurement apparatus causes a perturbation at length scales comparable to the size of the object we have to live with the fact that averages of the initial conditions are all we will ever know, even if the particles have themselves a deterministic behaviour. This line of thought leads us to the speculation that Schrödinger's equation contains within itself the statistics of the measurement apparatus.

\section{Appendix A}

Here we present the first few orders of the perturbation theory that produces the classical limit. For simplicity, we present the calculation in the one-dimensional case. It is readily extended to higher dimensions.

The starting point is the one-dimensional version of the integrand of equation (7), where we substitute the identity (11) 


$$
\begin{aligned}
\lim _{y \rightarrow 0} \frac{\eta}{\mathrm{i}} \frac{\partial \hat{f}}{\partial t}-\frac{\eta^{2}}{m} & \frac{\partial}{\partial x} \frac{\partial \hat{f}}{\partial y}+\left[V\left(x+\frac{y}{2}\right)-V\left(x-\frac{y}{2}\right)\right. \\
& \left.-2 \sum_{n=1} \frac{1}{(2 n+1) !} \frac{\partial^{2 n+1} V}{\partial x^{2 n+1}}\left(\frac{y}{2}\right)^{2 n+1}\right] \hat{f}=0 .
\end{aligned}
$$

The term with the sum is the same one first calculated by Wigner [2]. Now, we propose for $\hat{f}$ an expansion of the form

$$
\hat{f}=\hat{f}_{0}+y^{4} \hat{f}_{4}+y^{5} \hat{f}_{5}+\cdots,
$$

which, when substituted into (A.1) produces the following hierarchy:

$$
\begin{aligned}
\mathcal{O}\left(y^{0}\right): \frac{\eta}{\mathrm{i}} \frac{\partial \hat{f}_{0}}{\partial t}-\frac{\eta^{2}}{m} \frac{\partial^{2} \hat{f}_{0}}{\partial x \partial y}+\left[V\left(x+\frac{y}{2}\right)-V\left(x-\frac{y}{2}\right)\right] \hat{f}_{0}=0 \\
\mathcal{O}\left(y^{3}\right): \frac{4 \eta^{2}}{m} \frac{\partial \hat{f}_{4}}{\partial x}=-\frac{1}{4(3 !)} \frac{\partial^{3} V}{\partial x^{3}} \hat{f}_{0} \\
\mathcal{O}\left(y^{4}\right): \frac{5 \eta^{2}}{m} \frac{\partial \hat{f}_{5}}{\partial x}=\frac{\eta}{\mathrm{i}} \frac{\partial \hat{f}_{4}}{\partial t}+\left[V\left(x+\frac{y}{2}\right)-V\left(x-\frac{y}{2}\right)\right] \hat{f}_{4} \\
\mathcal{O}\left(y^{5}\right): \frac{6 \eta^{2}}{m} \frac{\partial \hat{f}_{6}}{\partial x}=\left[V\left(x+\frac{y}{2}\right)-V\left(x-\frac{y}{2}\right)\right] \hat{f}_{5}+\frac{\eta}{\mathrm{i}} \frac{\partial \hat{f}_{5}}{\partial t}-\frac{1}{16(5 !)} \frac{\partial^{5} V}{\partial x^{5}} \hat{f}_{0} \\
\mathcal{O}\left(y^{6}\right): \frac{7 \eta^{2}}{m} \frac{\partial \hat{f}_{7}}{\partial x}=\frac{\eta}{\mathrm{i}} \frac{\partial \hat{f}_{6}}{\partial t}+\left[V\left(x+\frac{y}{2}\right)-V\left(x-\frac{y}{2}\right)\right] \hat{f}_{6} \\
\mathcal{O}\left(y^{7}\right): \frac{8 \eta^{2}}{m} \frac{\partial \hat{f}_{8}}{\partial x}=\left[V\left(x+\frac{y}{2}\right)-V\left(x-\frac{y}{2}\right)\right] \hat{f}_{7}+\frac{\eta}{\mathrm{i}} \frac{\partial \hat{f}_{7}}{\partial t} \\
-\frac{1}{2^{6}(7 !)} \frac{\partial^{7} V}{\partial x^{7}} \hat{f}_{0}-\frac{1}{2^{2}(3 !)} \frac{\partial^{3} V}{\partial x^{3}} \hat{f}_{4} \cdot
\end{aligned}
$$

Note that the left-hand side of the equations for the successive orders is very simple. However, to take the limit $y \rightarrow 0$, it is necessary to introduce the usual change of variables (9), and because of this the right-hand side is separable in $x^{\prime}$ and $x^{\prime \prime}$ only for the zeroth order.

\section{Appendix B}

In this appendix we calculate the average of a mixed operator in Fourier space. Let $B(x, p, t)$ be an analytic function of the variables $x$ and $p_{x} \equiv p$. Then $B(x, p, t)=\sum_{n, m} B_{n m} x^{n} p^{m}$, where we have abbreviated $B_{n m}(t)=B_{n m}$. The standard average of $B$ reads

$$
\langle B(x, p, t)\rangle=\frac{\int \sum_{n, m} B_{n m} x^{n} p^{m} f(x, p, t) \mathrm{d} x \mathrm{~d} p}{\int f(x, p, t) \mathrm{d} x \mathrm{~d} p} .
$$

Introducing $f$ written as a function of $\hat{f}$ as in equation (5), we obtain for the integral in the numerator the expression

$$
\begin{aligned}
\int x^{n} p^{m} f \mathrm{~d} x \mathrm{~d} p & =\frac{1}{2 \pi \eta} \int \mathrm{d} x \mathrm{~d} p x^{n} p^{m} \int \mathrm{d} y \mathrm{e}^{-\frac{1}{\eta} p y} \hat{f}(x, y, t) \\
& =\int \frac{\mathrm{d} x \mathrm{~d} p}{2 \pi \eta} x^{n}\left(\frac{\eta}{\mathrm{i}}\right)^{m} \int \mathrm{d} y \mathrm{e}^{-\frac{\mathrm{i}}{\eta} p y} \frac{\partial^{m} \hat{f}}{\partial y^{m}}
\end{aligned}
$$

where we have integrated by parts $m$ times to obtain the last expression. The integral over $p$ reduces to a delta function in $y$, and $\left(\partial^{m} \hat{f} / \partial y^{m}\right)$ can be replaced by its expression as a function 
of $\psi$ and $\psi^{\dagger}$

$$
\begin{aligned}
\frac{\partial^{m} \hat{f}}{\partial y^{m}} & =\frac{\partial^{m}}{\partial y^{m}}\left[\psi^{\dagger}\left(x+\frac{y}{2}\right) \psi\left(x-\frac{y}{2}\right)\right] \\
& =\frac{1}{2^{m}} \sum_{k=0}^{m}(-1)^{k}\left(\begin{array}{l}
m \\
k
\end{array}\right) \frac{\partial^{k} \psi^{\dagger}}{\partial x^{\prime k}} \frac{\partial^{m-k} \psi}{\partial x^{\prime \prime m-k}},
\end{aligned}
$$

where $x^{\prime}$ and $x^{\prime \prime}$ are given by the one-dimensional version of (9). Then, the average reduces to

$$
\int x^{n} p^{m} f \mathrm{~d} x \mathrm{~d} p=\int \mathrm{d} x x^{n} \int \mathrm{d} y \delta(y)\left(\frac{\eta}{\mathrm{i}}\right)^{m} \frac{1}{2^{m}} \sum_{k=0}^{m}(-1)^{k}\left(\begin{array}{c}
m \\
k
\end{array}\right) \frac{\partial^{k} \psi^{\dagger}}{\partial x^{\prime k}} \frac{\partial^{m-k} \psi}{\partial x^{\prime \prime m-k}} .
$$

Integrating over $y$ sets $x^{\prime}=x^{\prime \prime}=x$. Once again we can integrate by parts $k$ times so that $\psi^{\dagger}$ appears as a multiplicative factor to the left

$$
\begin{aligned}
\int x^{n} p^{m} f \mathrm{~d} x \mathrm{~d} p & =\int \mathrm{d} x \psi^{\dagger} \frac{1}{2^{m}} \sum_{k=0}^{m}\left(\begin{array}{c}
m \\
k
\end{array}\right)\left(-\mathrm{i} \eta \frac{\partial}{\partial x}\right)^{k}\left(x^{n}\left(-\mathrm{i} \eta \frac{\partial}{\partial x}\right)^{m-k} \psi\right) \\
& =\int \mathrm{d} x \psi^{\dagger}\left[\frac{1}{2^{m}} \sum_{k=0}^{m}\left(\begin{array}{c}
m \\
k
\end{array}\right) \hat{p}^{k} \hat{x}^{n} \hat{p}^{m-k}\right] \psi,
\end{aligned}
$$

where we have introduced the notation $\hat{x}=x$ and $\hat{p}=-\mathrm{i} \eta(\partial / \partial x)$. Finally, replacing expression (B.5) into (B.1) and making use of the identity $\int f \mathrm{~d} x \mathrm{~d} p=\int \psi^{\dagger} \psi \mathrm{d} x$, we obtain

$$
\begin{aligned}
\langle B(x, p)\rangle & =\frac{\int B(x, p) f(x, p, t) \mathrm{d} x \mathrm{~d} p}{\int f(x, p, t) \mathrm{d} x \mathrm{~d} p} \\
& =\frac{\int \psi^{\dagger} \hat{B}_{H}(\hat{x}, \hat{p}) \psi \mathrm{d} x}{\int \psi \psi^{\dagger} \mathrm{d} x}
\end{aligned}
$$

where

$$
\hat{B}_{H}=\sum_{n, m} B_{n m} \frac{1}{2^{m}} \sum_{k=0}^{m}\left(\begin{array}{l}
m \\
k
\end{array}\right) \hat{p}^{k}\left(\hat{x}^{n} \hat{p}^{m-k}\right) .
$$

The quantity $\hat{B}_{H}$ is now a Hermitian operator. This last expression can also be written as

$$
\hat{B}_{H}=\sum_{n, m} B_{n m} \frac{1}{2^{n}} \sum_{k=0}^{n}\left(\begin{array}{l}
n \\
k
\end{array}\right) \hat{x}^{n-k}\left(\hat{p}^{m} \hat{x}^{k}\right)
$$

To show this we go back to equation (42). If instead of directly integrating over $y$ we perform the change of variables (9)

$$
\int x^{n} p^{m} f \mathrm{~d} x \mathrm{~d} p=\int \mathrm{d} x^{\prime} \mathrm{d} x^{\prime \prime} \delta\left(x^{\prime}-x^{\prime \prime}\right)\left(\frac{x^{\prime}+x^{\prime \prime}}{2}\right)^{n}\left(\frac{\eta}{\mathrm{i}}\right)^{m} \frac{1}{2^{m}} \sum_{k=0}^{m}(-1)^{k}\left(\begin{array}{c}
m \\
k
\end{array}\right) \frac{\partial^{k} \psi^{\dagger}}{\partial x^{\prime k}} \frac{\partial^{m-k} \psi}{\partial x^{\prime \prime m-k}} .
$$

Using the binomial expansion of $\left(x^{\prime}+x^{\prime \prime}\right)^{n}$ and the fact that $x^{\prime \prime n-j}\left(\partial^{k} \psi^{\dagger} / \partial x^{\prime k}\right)=$ $\partial^{k}\left(x^{\prime \prime n-j} \psi^{\dagger}\right) / \partial x^{\prime k}$ and also $x^{\prime j}\left(\partial^{m-k} \psi^{\dagger} / \partial x^{\prime m-k}\right)=\partial^{m-k}\left(x^{\prime \prime j} \psi^{\dagger}\right) / \partial x^{\prime m-k}$ we can rewrite the average as the double sum

$$
\begin{aligned}
& \int x^{n} p^{m} f \mathrm{~d} x \mathrm{~d} p=\int \mathrm{d} x^{\prime} \mathrm{d} x^{\prime \prime} \delta\left(x^{\prime}-x^{\prime \prime}\right)\left(\frac{\eta}{\mathrm{i}}\right)^{m} \sum_{k, j=0} \frac{(-1)^{k}}{2^{n+m}} \\
& \times\left(\begin{array}{l}
m \\
k
\end{array}\right)\left(\begin{array}{l}
n \\
j
\end{array}\right) \frac{\partial^{k}}{\partial x^{\prime k}}\left(x^{\prime \prime n-j} \psi^{\dagger}\right) \frac{\partial^{m-k}}{\partial x^{\prime \prime m-k}}\left(x^{\prime j} \psi\right) .
\end{aligned}
$$


Now we can perform the integral over $x^{\prime}$ which sets $x^{\prime}=x^{\prime \prime} \equiv x$. After integrating $k$ times by parts and replacing $\sum_{k=0}^{n}\left(\begin{array}{c}m \\ k\end{array}\right)=2^{m}$, we obtain

$$
\begin{aligned}
\int x^{n} p^{m} f \mathrm{~d} x \mathrm{~d} p & =\int \mathrm{d} x \psi^{\dagger} \frac{1}{2^{n}} \sum_{j=0}^{n}\left(\begin{array}{c}
n \\
j
\end{array}\right) x^{n-j}(-\mathrm{i} \eta)^{m} \frac{\partial^{m}}{\partial x^{m}}\left(x^{j} \psi\right) \\
& =\int \mathrm{d} x \psi^{\dagger}\left[\frac{1}{2^{n}} \sum_{j=0}^{n}\left(\begin{array}{l}
n \\
j
\end{array}\right) \hat{x}^{n-j} \hat{p}^{m} \hat{x}^{j}\right] \psi .
\end{aligned}
$$

The equality of the terms in between the square brackets of (B.5) and (B.11) was first proven by McCoy [23].

\section{References}

[1] Bohm D 1989 Quantum Theory (New York: Dover)

[2] Wigner E 1932 Phys. Rev. 40 749-59

[3] Wagner R, Peverly P, Su Q and Grobe R 2000 Phys. Rev. A 61035402

[4] Kim H, Lee M, Yi J and Kim J 1996 Phys. Rev. A 53 3767-72

[5] Zurek W H 2003 Rev. Mod. Phys. 75 715-75

[6] Zurek W H 1991 Phys. Today 44 36-44

[7] Guilini D, Joos E, Kiefer C, Kupsch J, Stamatescu I O and Zeh H D 1996 Decoherence and the Appearance of a Classical World in Quantum Theory (New York: Springer)

[8] Chapman M S, Hammond T D, Lenef A, Schmiedmayer J, Rubenstein R R, Smith E and Pritchard D E 1995 Phys. Rev. Lett. 75 3783-6

[9] Kokorowski D A, Cronin A D, Roberts T and Pritchard D 2001 Phys. Rev. Lett. 86 2191-4

[10] Hornberger K, Uttenthaler S, Brezger B, Hackermuller L, Arndt M and Zeilinger A 2003 Phys. Rev. Lett. 90160401

[11] Hackermuller L, Hornberger K, Brezger B, Zeilinger A and Arndt M 2004 Nature 427 711-4

[12] Madelung E 1926 Z. Phys. 40 322-6

[13] Takabayasi T 1955 Prog. Theor. Phys. 14 238-302

[14] Halbwachs F 1960 Théorie Relativiste des Fluids à Spin (Paris: Gauthier-Villars)

[15] Pesci A I and Goldstein R E 2005 Nonlinearity 18 211-26

[16] Pesci A I, Goldstein R E and Uys H 2005 Nonlinearity 18 227-35

[17] Pesci A I, Goldstein R E and Uys H 2005 Nonlinearity 18 1295-304

[18] Liboff R L 1998 Kinetic Theory: Classical, Quantum and Relativistic Descriptions (New York: WileyInterscience)

[19] Fröhlich H 1967 Physica 37 215-26

[20] Fröhlich H 1973 Riv. Nuovo Cimento 3 490-534

[21] Kreuzer H J 1981 Nonequilibrium Thermodynamics and its Statistical Foundations (New York: Oxford University Press)

[22] Kaniadakis G 2002 Physica A 307 172-84

[23] McCoy N H 1932 Proc. Natl Acad. Sci. USA 18 674-6

[24] Weyl H 1927 Z. Phys. 46 1-46

[25] Shewell J R 1959 Am. J. Phys. 27 16-21

[26] Cohen L 1970 J. Math. Phys. 11 3296-7

[27] Dowker J S 1976 J. Math. Phys. 17 1873-4

[28] Born M and Jordan P 1925 Z. Phys. 34 858-88 